\title{
Investigation of the Force Vectors Acting on the Potato in the Clamping Finger Type Dosing System of a Potato Planter
}

\author{
Szilárd ILYÉS, ${ }^{1}$ Judit PÁSZTOR ${ }^{2}$ \\ ${ }^{1}$ Sapientia Hungarian University of Transylvania, Faculty of Technical and Human Sciences, \\ Department of Mechanical Engineering, Târgu Mureș, Romania, ilyes.szilard@ms.sapientia.ro \\ ${ }^{2}$ Sapientia Hungarian University of Transylvania, Faculty of Technical and Human Sciences, \\ Department of Mechanical Engineering, Târgu Mureș, Romania, pjudit@ms.sapientia.ro
}

\begin{abstract}
The operational accuracy of the dosing system of the potato planting machine influences the planting plant spacing, and indirectly the crop yield. The operating accuracy depends on the design of the dosing system, its kinematics and the forces acting on the planting tubers. This dissertation presents the force vectors acting on the potato in the dosing system of the guided clamping finger type potato planting machine and their spatial motion during the dosing process. Examination of the vectors reveals the improved possibilities of tuber fixation.
\end{abstract}

Keywords: potato planter, clamping-fingers, guide rail, dosing device, vector.

\section{Introduction}

Agricultural engineering, which serves the agricultural sciences, clearly contributes to the improvement of the work quality parameters of existing machines by developing technical solutions that often seem simple.

The potato planter with a clamping finger dosing system has become widespread in Eastern Europe, thanks to the former Czechoslovakia industry.

The construction and operation of the clamping-finger type dosing device of potato planter is illustrated in Figure 1 [1, 2]. Planting disc 1 makes a circular motion, getting its drive from the ground-wheel drive wheel through chain drives and a Norton transmission. Usually 12 clamping-fingers 2 are attached to the planting disc. The clamping devices rotate around their axes 3 , against the torsion spring 4 . The rotation is controlled by cam 5 , so the clamping finger fixes the tuber 7. Control is performed by guide rail 6 .

The guide rail closes the clamping fingers as it sinks into the tuber container, and opens them upwards. The finger clamps and lifts the tubers. It transports them until the guide rail opens again above the down comer. This process can be fol-

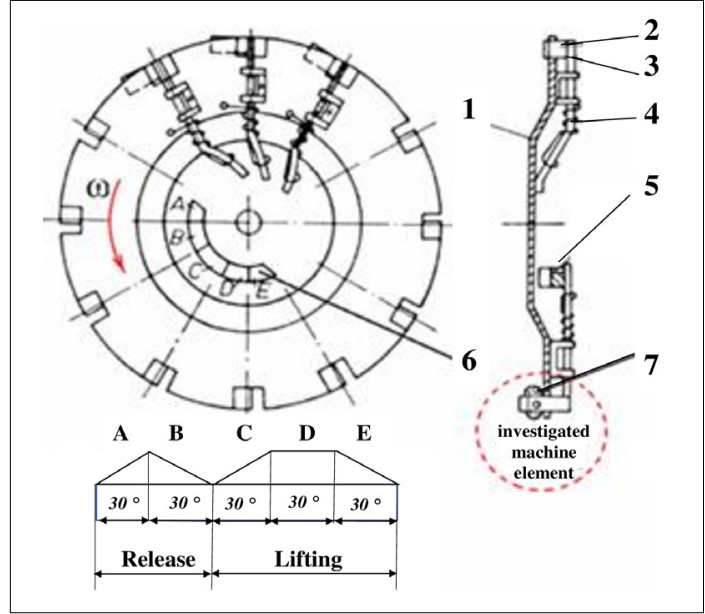

Figure 1. Clamping-finger type dosing device. [1]

lowed in Figure 1. The tuber is placed in the furrow opened by the coulter.

\section{Analysis of the position of force vec- tors acting on tubers during rotation}

The force vectors acting on the $C_{M}$ mass-centred tubers can be followed in Figure 2. In position 1 the finger clamps the tuber, in positions 1-2-3 
the tuber is transported. In position 3, the finger releases the tuber. In position 4 the finger closes.

The following simplifications are used in the study [3]:

- The tuber describes a complete circle on the disc, but in reality, the sections 3-4 are omitted (Figure 1).

- The disc is considered a flat surface [4], the air resistance is neglected.

- The tubers are considered spherical.

In the $\mathrm{O}_{\mathrm{d}} \mathrm{XYZ}$ rectangular coordinate system, the resultant force vector $\mathbf{R}$, characterized by radius $r$ and by rotation angle $\phi=\phi(t)[\mathrm{rad}]$ concentrated in $C_{M}$ pontba koncentrált $\mathbf{R}$ concentrated in $\mathbf{G}$, the centrifugal force $\mathbf{F}_{\mathbf{c}}$ and the normal force $\mathbf{F}_{\mathbf{p}}$ :

$$
\begin{aligned}
& \mathbf{R}=\mathbf{G}+\mathbf{F}_{\mathbf{c}}+\mathbf{F}_{\mathbf{p}}[\mathrm{N}], \\
& \mathbf{G}=m \cdot g[\mathrm{~N}], \\
& \mathbf{F}_{\mathbf{c}}=m \omega^{2} r[\mathrm{~N}], \\
& \mathbf{F}_{\mathbf{p}}=k \cdot \theta / l[\mathrm{~N}],
\end{aligned}
$$

where: $m$ is the weight of the tuber $[\mathrm{kg}]$;

$g$ gravitational acceleration $\left[\mathrm{m} / \mathrm{s}^{2}\right]$;

$\omega$ angular velocity $[\mathrm{rad} / \mathrm{s}]$;

$r$ is the distance of the $C_{M}$ points from the point of rotation [m];

$k$ is the torsion spring characteristic [m/rad];

$\theta$ opening angle of the clamping finger $\left[^{\circ}\right][5]$;

$l$ is the length of the clamping finger between the axis of rotation and the point where the potato relies on $[\mathrm{m}]$.

The gravitational force $\mathbf{G}$ depends on the tuber mass $\mathrm{m}\left(d_{t}\right)$, indirectly on the tuber diameter $d_{t}$.

The centrifugal force $\mathbf{F}_{\mathbf{c}}$ depends on the tuber mass $\mathrm{m}\left(d_{t}\right)$ and the angular velocity.

The normal force $\mathbf{F}_{\mathbf{p}}$ (Figure 3) with the clamping finger 2 it presses the tuber 3 on the plane of the vertical disc 1.

In each of the correlations (1-4) the diameter $d_{t}$ of the tuber (Figure 2) influences the bite angle $\theta$ of the clamping fingers. The tuber diameter can be considered constant for one rotation, thus the

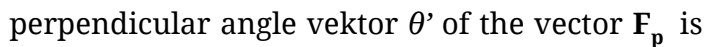
also constant, its value is between $0<\theta^{\prime}<\pi / 2$. In practice, the bite angle $\theta$ of the clamping finger can be considered smaller than the maximum theoretical value $\pi / 2$.

At any position $i=\overline{1,4}$ of $C_{M}$ (eg. $\mathrm{i}=2$, if $\phi=\pi / 2$ ), the vector $\mathbf{G}$ and the variable axes $C_{M} Z_{i}$ define the parallel planes $\Phi_{i}$ (yellow) (Figure 2) [6]. The clamping finger 2 (Figure 3 ) and its projection on the disc 1 determine the plane of rotation $\Delta$ (red) (Figure 2). The forces $\mathbf{F}_{\mathbf{c}}$ and $\mathbf{G}$ define the $\Gamma$ (grey)

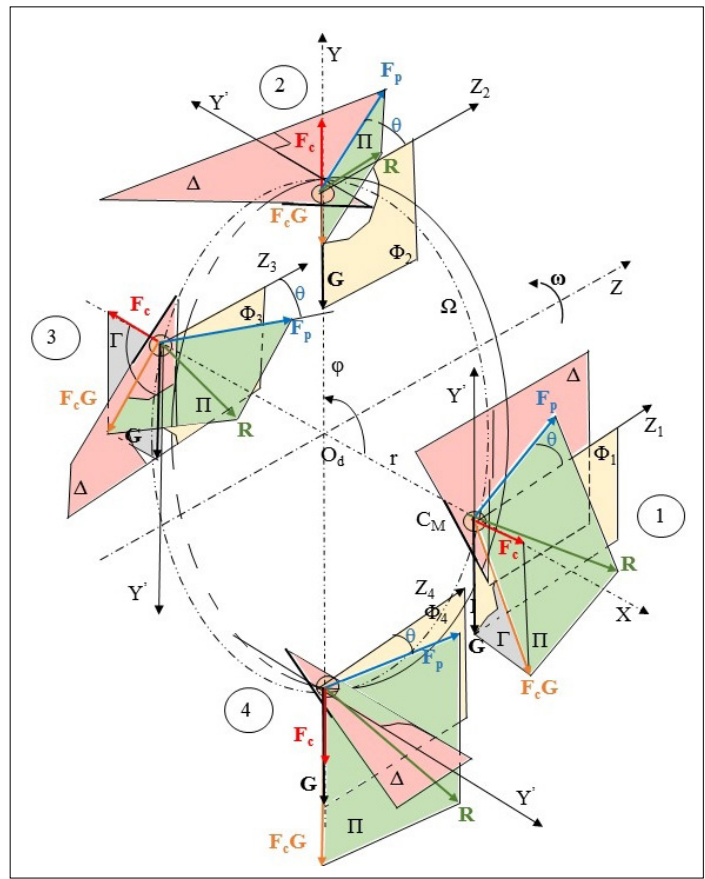

Figure 2. Plane representation of the forces acting at $C_{M}$ point.

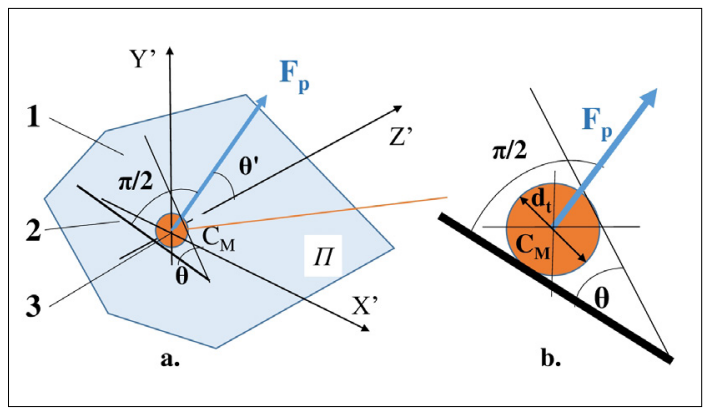

Figure 3. The effect of the clamping finger in the $C_{M}$ point.

plane, which is parallel to the $\mathrm{O}_{\mathrm{d}} \mathrm{XY}$ disk plane. The result of the $\mathbf{F}_{\mathbf{c}}$ and $\mathbf{G}$ forces is the $\mathbf{F}_{\mathbf{c}} \mathbf{G}$ force. The vectors $\mathbf{F}_{\mathbf{c}} \mathbf{G}$ and $\mathbf{F}_{\mathbf{p}}$ define the $\Pi$ (green) plane, resulting in the vector $\mathbf{R}$. The vector $\mathbf{R}$ is directed from the clamping finger to the disc, its size and direction depending on the factors of the other three vectors.

The result of $\mathbf{F}_{\mathbf{c}} \mathbf{G}$ is given by equation (5):

$$
F_{C G}=\sqrt{F_{c}^{2}+G^{2}+2 F_{c} G \cos \left(\varphi+\frac{\pi}{2}\right)} \text {. }
$$

The normal force vector $\mathbf{F}_{\mathbf{p}}$ of the clamping finger is included in the $\Delta$ (red) plane, its value is constant, its direction is perpendicular to the $\Gamma$ (grey) plane. 
The projection $\mathbf{F}_{\mathbf{p}} \sin \theta$ of the normal force $\mathbf{F}_{\mathbf{p}}$ on the plane of the disc at any $C_{M}$ point perpendicular to the radius (Figure 4). The projection vector forms a constant angle $\alpha$ with the radius belonging to the point $C_{M}(6)$ :

$$
\alpha=\operatorname{arctg} \frac{F_{p} \sin \theta}{r}
$$

Since both $\mathbf{F}_{\mathbf{c}} \mathbf{G}$ and direction of $\mathbf{F}_{\mathbf{p}}$ depend on $\phi$ (Figure 2), both the resulting $\mathbf{R}$ is a function of $\phi$.

Examining the forces $\mathbf{G}, \mathbf{F}_{\mathbf{c}}, \mathbf{F}_{\mathbf{p}}$ acting on the $C_{M}$ points according to the $\mathrm{OZ}$ axis of rotation, the following rotation bodies are obtained in the case of circular motion:

- an annulus, a flat ring, varying in thickness between 0 and $G$ (Figure 5a); ,

- an annulus, a flat ring with a constant thickness $F_{c}$ (Figure 5b);

- a concave-shaped annular body of rotation with $\rho$ curve (Figure 5c), with radiuses $r$ and $O_{d}{ }^{\prime} A_{1}$, height $F_{p} \cos \theta$, (Figure 4). Relationship between radiuses:

$$
o_{d}^{\prime} A_{1}=\sqrt{r^{2}+\left(F_{p} \sin \theta\right)^{2}}
$$

The opening of the angle $\theta$ does not reach the limit cases due to the construction of the clamping finger and the minimum tuber diameter.

The rotation body of the vector $\mathbf{R}$ is a complex spatial shape, since both its size and the angle of the $\Pi$ (green) plane with the disk vary due to the change in the force vectors $\mathbf{F}_{\mathbf{c}} \mathbf{G}$ (Figure 3).

\section{Analysis of the position of force vectors acting on tubers projected in one point}

The position of the force vectors $\mathbf{G}, \mathbf{F}_{\mathbf{c}}, \mathbf{F}_{\mathbf{p}}$ acting on $C_{M}$ mass-centred tubers is shown in Figures 6 . and 7.

The force vectors $\mathbf{F}_{\mathbf{p}}$ create a cone mantle with radius $F_{p} \sin \theta$ around a $\mathrm{C}_{\mathrm{M}} \mathrm{Z}^{\prime}$ axis parallel to $\mathrm{O}_{\mathrm{d}} \mathrm{Z}$ (Figure 6) .

The vector $\mathbf{G}$ is always vertical.

The $\mathbf{F}_{\mathbf{c}}$ vectors form a solid (red) circle of radius $F_{c}$ (Figure 7).

For a constant tuber diameter $d_{t}$ the position of the normal force vectors $\mathbf{F}_{\mathbf{p}}$ is shown in Figure 6 .

It can be seen that the vertices of the resulting $\mathbf{F}_{\mathbf{c}} \mathbf{G}$ vectors describe a $C_{R}$ circle also with $F_{c}$ íradius, the centre of which is at the vertex of the $\mathbf{G}$ vector (Figure 7).

By rotating the parallelogram of $\mathrm{O}_{\mathrm{t}} \mathrm{GF}_{c} \mathrm{G}_{\pi} \pi$ around the $O_{t} G$ side, the offsets of $\mathbf{F}_{\mathbf{c}}$ are created by shifts parallel to the $O_{t} G$ side. According to the magnitude of $\mathbf{G}$ the mutual position of the circle

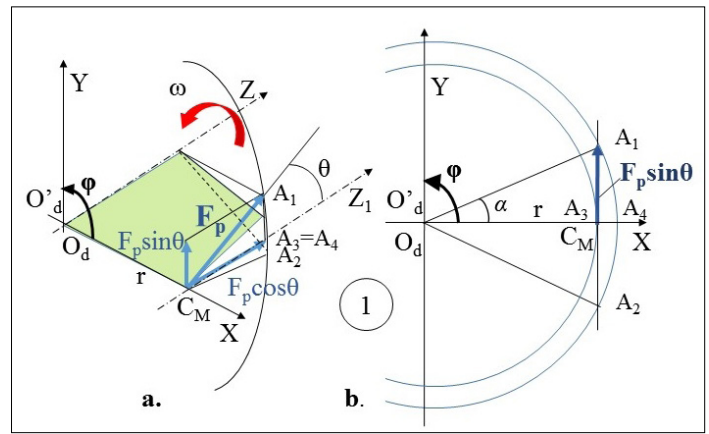

Figure 4. Calculation of $\mathbf{F}_{\mathbf{p}}$ in position 1.

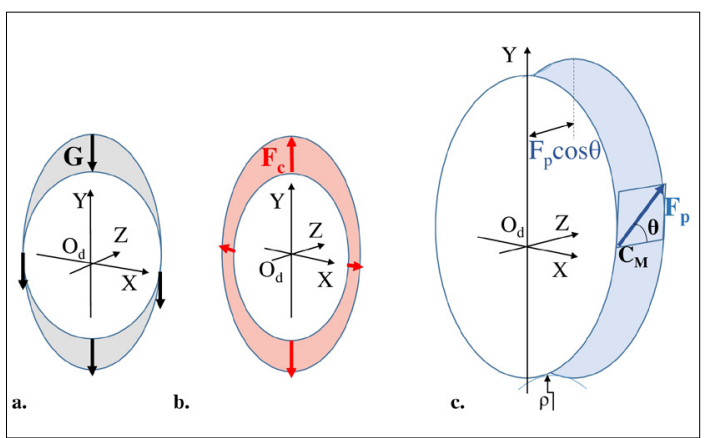

Figure 5. Rotation bodies of $\mathbf{G}, \mathbf{F}_{\mathbf{c}}, \mathbf{F}_{\mathbf{p}}$.

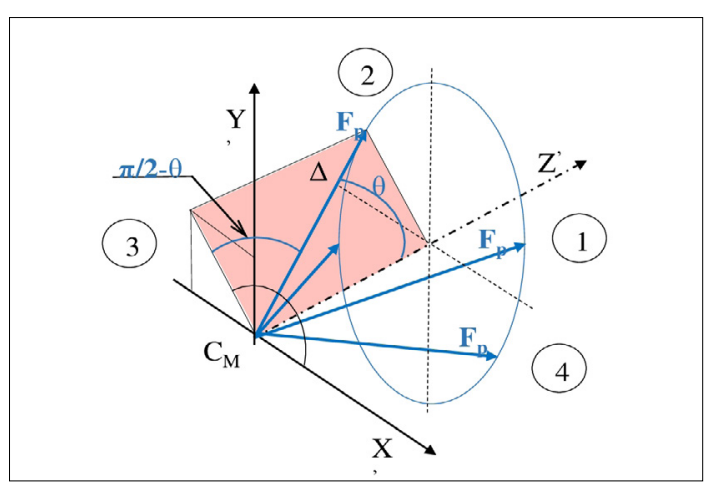

Figure 6. The shape of the mantle $\mathbf{F}_{\mathbf{p}}$ projected into the $C_{M}$ point.

$\mathrm{C}_{\mathrm{Fc}}$ and $\mathrm{C}_{\mathrm{R}}$ determines the spread surface of the force resulting from $\mathbf{F}_{\mathbf{c}} \mathbf{G}$.

Analysing the planar and spatial graphical forms of the force vectors, it can be noticed that the values and directions of the variable vectors concentrated at any $C_{M}$ point cannot be arbitrarily changed in order to better retain the tuber.

Reducing the angle $\theta$ ' of the normal force $\mathbf{F}_{\mathbf{p}}$ can help keep the tuber better. This can be done by improving the geometric shapes of the support elements, the $l$ length of the support clamping finger and the spring characteristics $k$. 


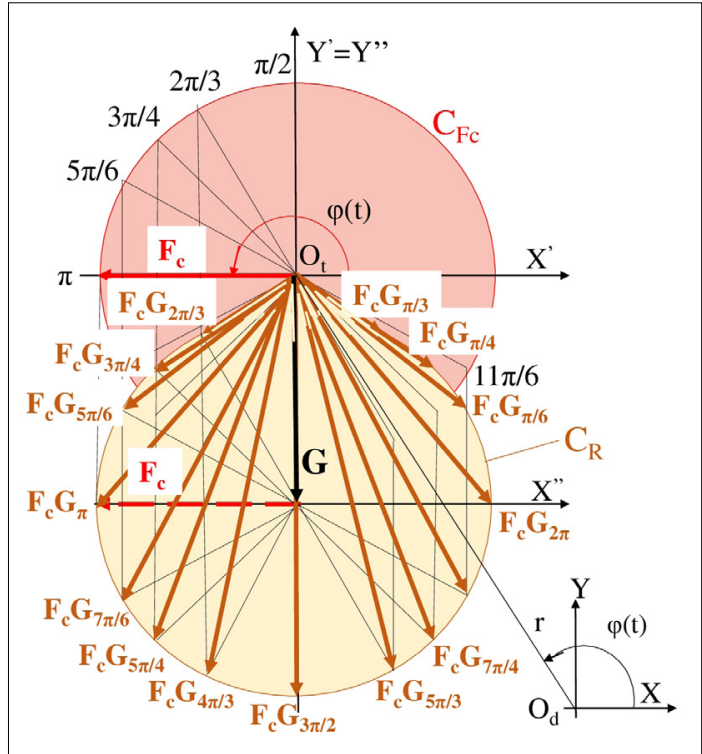

Figure 7. Representation of $\mathbf{F}_{\mathbf{c}}$ és $\mathbf{G}$ acting at $C_{M}$

In the case of a curved finger, the bite angle $\theta=m\left(Z^{\prime} \widehat{C_{M} F} p\right)$ (Figure 7) decreases continuously along the length of the finger. Thus, at any point of contact with the tuber, the deflection angle $\theta$ ' of the normal force $\mathbf{F}_{\mathbf{p}}$ will be smaller than the original bite angle $\theta$ of the clamping finger (Figure 3).

Additional possibilities for tuber retention: by forming the curved clamping finger into a spoon shape, the scattering in the variable direction of the resulting vector $\mathbf{R}$ acting on the $C_{M}$ point can be taken into account.

\section{Analysis of the position of force vectors during rotation for different tuber diameters}

During the dosing operation, the diameter $d_{t}$ of the tubers are different. The maximum value of the bite angle $\theta$ of the clamping finger is $\pi / 2$. Its minimum value is determined by the smallest $d_{t}$ tuber diameter. In this case, the expansion of the force vectors $\mathbf{F}_{\mathbf{p}}$ in the $\mathrm{X}^{\prime} \mathrm{O}_{\mathrm{t}} \mathrm{Z}^{\prime}$ plane describes the $\mathrm{F}_{\mathrm{p} 2} \mathrm{O}_{\mathrm{t}} \mathrm{F}_{\mathrm{p} 3}$ circular area (Figure 8.b). During the rotation of the $\mathbf{F}_{\mathbf{p}}$ ' vector around $\mathrm{O}_{\mathrm{d}} \mathrm{Z}$ the $O_{t}$ ' point moves to the $O_{t}$ position, the $F_{p 1}$ point to the $F_{p 2}$ position, and the $F_{p 3}$ vector performs translation, determining the annular body of rotation $T\left(F_{p 1} O_{t} F_{p 3}\right)$. From the triangles $\mathrm{F}_{p 2} \mathrm{O}_{t} \mathrm{O}_{d}$ and $\mathrm{FF}_{p 1} \mathrm{O}_{d}$ the maximum thickness $d$ of the rotation body can be determined:

$$
d=\sqrt{r^{2}+F_{p}^{2}}-r
$$

The arc of $\mathrm{C}_{\mathrm{Fp}}\left(\mathrm{F}_{\mathrm{p} 2}, \mathrm{O}_{\mathrm{t}}, \mathrm{F}_{\mathrm{p} 3}\right)$ with radius $F_{p}$ is a quarter of a complete circle of $\mathrm{C}_{\mathrm{Fp}}\left(\mathrm{O}_{\mathrm{t}}, \mathrm{F}_{\mathrm{p}}\right)$ in the $\mathrm{X}^{\prime} \mathrm{O}_{\mathrm{t}} \mathrm{Z}^{\prime}$ plane. The plane of this circle is at distance $r$ at point $O_{t}$ in a sphere of radius $r+d$ with centre $O_{d}$ (Figure 8.a). Parts of this sphere are $\left(F_{p 1} F_{p 2}\right)$, $\left(F_{p 1} F_{p 3}\right)$ and $\left(F_{p 2} F_{p 3}\right)$ quarter-circles, which are also parts of a spherical cap of height $d$. Thus, the three curves define the quarter sphere of radius $F_{c}$ (Figure 8.b). The centre of the forming spherical cap is $O_{d}$, its radius is $r+d$, the radius of its intersecting circle is $\mathbf{F}_{\mathbf{c}}$, and its height is $d$. Thus, the body of rotation of the vector $\mathbf{F}_{\mathbf{c}}$ is a ring, defined

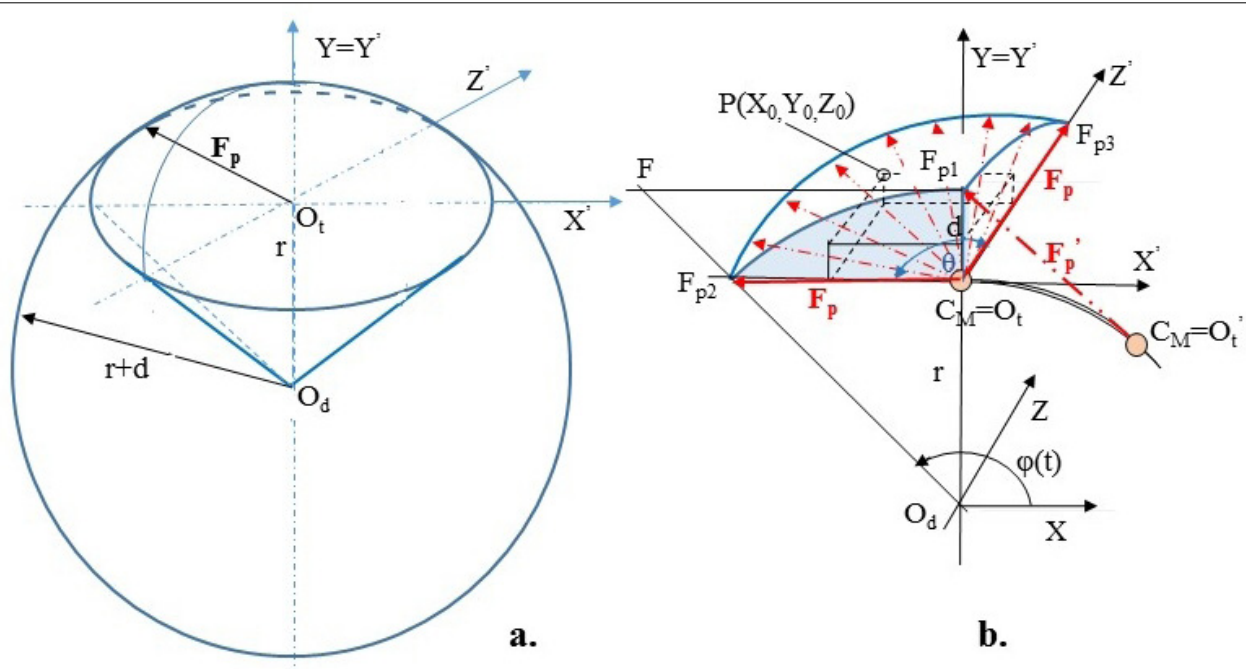

Figure 8. Rotation body formed by $\mathbf{F}_{\mathbf{p}}$ at variable $d_{t}$. 
by a quarter of a spherical cap of radius $r+d$. The generatrixes are the circular arc $F_{p 1} F_{p 2}$, the maximum thickness $O_{d} F_{p 1}=d$ and the height $O_{t} F p_{3}$. The generatrixes rotate around the $\mathrm{O}_{\mathrm{d}} \mathrm{Z}$ axis with radius $r$.

The equation of the generatrix curve is the equation of a circular arc of radius $r+d$ drawn from $O_{d}(9)$, the length of which is delimited by $F_{p 1}$ and $F_{p 3}(10)$ :

$$
Y^{2}+Z^{2}=(r+d)^{2} \text {, }
$$

where:

$$
X=0, \quad r<Y<r+d, \quad 0<z<F p_{3} .
$$

\section{Conclusions}

A graphical study of the force vectors acting on the clamping finger planting structure provides a picture of the planar or spatial changes of the force vectors and illustrates the operating limits of the dosing device.

The magnitude of the force vectors acting on the tuber depends on the tuber mass and indirectly on tuber diameter. The magnitude of the resultant force also depends on the angle of rotation of the planting disc.

The force vectors $\mathbf{F}_{\mathbf{p}}$ are projected into the $C_{M}$ mass-centre of the tuber, they form a straight conical rotation body with an angle $\theta^{\prime}$.

The resulting $\mathbf{R}$ vector forms a complex rotation body over one entire cycle due to their varying direction and magnitude. Its representation is difficult without a computer graphic method.

The clamping between the finger and the disc depends on the tuber size, the angular velocity of the disc, the geometry of the clamping device and the normal force $\mathbf{F}_{\mathbf{p}}$ With a good design of the geometry of the clamping finger, in addition to the constant normal force, by changing the direction of the vector, a better fixation of the tuber could be achieved. Thus, in order to improve the tuber fixation, it is recommended to bend the finger.

The changing direction and size of the resulting $\mathbf{R}$ vector justifies the spooning and rinsing of the clamping finger in order to fix the tubers more securely.

The study points to the development possibilities of fixing tubers on the guide track clamping finger potato planting system.

\section{References}

[1] Căproiu Ş., Sripnic V., Ciubotaru C., Babiciu P., Roș V.: Maşini agricole de lucrat solul, semănat şi întreţinerea culturilor. Editura Didactică şi Pedagogică, București, 1982.

[2] Zheng Z., Zhao H., Liu Z., He J., Liu W.: Research Progress and Development of Mechanized Potato Planters: A Review. Agriculture, 11/6. (2011) 521. https://doi.org/10.3390/agriculture11060521

[3] Rus F.: Mașini agricole pentru lucrările solului, semănat și intreținerea culturilor. Editura Universitatea Transilvania Brașov, Brassó, 1975.

[4] Ilyés Sz:: A szorítóujjas rögzítés geometriai és dinamikai elemzése a burgonyaültető gép esetében. XXVIII. Nemzetközi Gépészeti Konferencia, Székelyudvarhely-online, Románia, OGÉT 2020, 2020. 105-108. https://ojs.emt.ro/index.php/oget/article/view/207

[5] Jula A., Lateș M.: Organe de mașini. Universitatea Transilvania Brașov Kiadó, Brassó, 2004.

[6] Máté M.: Müszaki mechanika - kinematika. EME Kiadó, Kolozsvár, 2010. https://doi.org/10.36242/mtf-10 